\title{
Role of Free Radicals, Glutamate Toxicity, Glutathione Depletion in Apoptosis of Cochlear Hair Cells, Neuronal Cells among Patients with Sensorineural Hearing Loss
}

\author{
Vithal D. Udagatti ${ }^{1}$ Rajendran Dinesh Kumar ${ }^{2, \odot}$ \\ ${ }^{1}$ N.R.M ENT Hospital, Raichur, Karnataka, India \\ ${ }^{2}$ Rajarajeswari Medical College and Hospital, Department of \\ ENT and Head-Neck Surgery, Bengaluru, Karnataka, India
}

\author{
Arjunsing Vijaysing Samorekar ${ }^{2}$ Vaibhavi KR ${ }^{2}$
}

\begin{abstract}
Address for correspondence Rajendran Dinesh Kumar, MBBS, MS ENT, Assistant Professor, Rajarajeswari Medical College and Hospital, Department of ENT and Head-Neck Surgery, Bengaluru 560074, Karnataka, India (e-mail: dinuraj1186@gmail.com).
\end{abstract}

\begin{abstract}
Keywords

- presbyacusis

- glutathione

- sensorineural

hearing loss

- free radicals

Introduction Hearing loss may lead to depression, decreased quality of life, reduced functional status and social isolation. The glutathione-S transferase (GSTS) is an antioxidant scavenging enzyme. Decreased glutathione and GSTS activity levels lead to an increase in susceptibility of hair-cell damage leading to sensorineural hearing loss. The cumulative effect of oxidative stress and mitochondrial damage by free radicals results in the mutation/deletion of deoxyribonucleic acid, leading to decline in mitochondrial function, which in turn plays an important role in inducing apoptosis of the cochlear cells. Other risk factors also include noise exposure, genetic predisposition, health comorbidities, ototoxic drugs, infections, and immune-mediated inflammation of auditory cells.

Study Design Prospective, non-comparative, metacentric clinical study.

Materials and Methods The study was carried out in 30 patients from 6/5/2016 to $10 / 1 / 2018$. Total of 30 patients of sensorineural hearing loss were enrolled ( 17 males, 13 females). Clinical history, ENT examination, and audiogram were done, treatment duration of 8 weeks for each patient and followed up to 3 outpatient visits. The patient was administered rebamipide $100 \mathrm{mg}$, alpha lipoic acid $100 \mathrm{mg}$, and acetylcysteine $100 \mathrm{mg}$ capsules twice a day for a total period of 8 weeks. Wherever giddiness was an added symptom, Cinnarizine $20 \mathrm{mg}$ with Diaminehydrate $40 \mathrm{mg}$ combination twice a day was added up to complete relief of symptoms, thereafter once a day as maintenance dose over a period of 8 weeks. Wherever tinnitus was an added symptom, deflazacort $6 \mathrm{mg}$ twice a day was added and tapered up to 1 month. If the symptom of tinnitus persisted, intratympanic steroid injection was given. During every visit, clinical assessment and audiogram were repeated.

Results Our study demonstrated greater improvement in hearing at higher frequencies with 8 weeks of rebamipide $100 \mathrm{mg}+$ alpha lipoic acid $100 \mathrm{mg}+$ acetylcysteine $100 \mathrm{mg}$ administration in 30 patients with twice-daily dosing.
\end{abstract}

DOI https://doi.org/

$10.1055 / \mathrm{s}-0041-1724220$

ISSN 2581-9607.
(C)2021. Indian Society of Otology.

This is an open access article published by Thieme under the terms of the Creative Commons Attribution-NonDerivative-NonCommercial-License, permitting copying and reproduction so long as the original work is given appropriate credit. Contents may not be used for commercial purposes, or adapted, remixed, transformed or built upon. (https://creativecommons.org/licenses/by-nc-nd/4.0/).

Thieme Medical and Scientific Publishers Pvt. Ltd. A-12, 2nd Floor, Sector 2, Noida-201301 UP, India 
Conclusion Synthesis of free radicals in the inner ear may play an important part in the pathogenesis of sensory hearing loss. The combination of rebamipide $100 \mathrm{mg}+$ alpha lipoic acid $100 \mathrm{mg}+$ acetylcysteine $100 \mathrm{mg}$ is effective prophylaxis in sensorineural hearing loss that addresses both factors of inhibiting the cochlear cell damage and enhancing cochlear cell preservation.

\section{Introduction}

The cumulative effect of oxidative stress and mitochondrial damage by free radicals results in accumulation of mutated deoxyribonucleic acid (DNA). Mutation/deletions causes decline in mitochondrial function that plays an important role in inducing apoptosis of the cochlear cells. ${ }^{1}$ Other risk factors include noise exposure, genetic predisposition, health comorbidities such as atherosclerosis, diabetes, high blood pressure and smoking. Exposure to ototoxic drugs, infection and immune-induced inflammation of auditory cells can also cause cochlear cell damage. $^{2}$

The glutathione-S transferase (GSTS) is an antioxidant that scavenges enzyme. Decreased glutathione and GSTS activity levels cause an increase in susceptibility of hair-cell damage leading to sensorineural hearing loss. ${ }^{3}$ The role of oxidative stress, free radicals, inflammation of cells of the cochlea, mitochondrial damage and reduction in glutathione enzyme in the development of sensorineural loss are well documented. Hearing loss may lead to depression, decreased quality of life, reduced functional status, and social isolation. , $^{4}$

\section{Anatomy}

The inner ear is divided into bony and membrane labyrinth, which lies within the petrous portion of the temporal bone. Bony labyrinth consists of a vestibule, semicircular canal, and cochlea. The cochlea is cone shaped with $23 / 4$ turn's and its base lies against the vestibule. Membrane labyrinth has vestibule (saccule, utricle), semicircular canals cochlea and endolymphatic sac. The cochlea is divided into scala tympani, scala media, and scala vestibuli. Scalamedia in membrane labyrinth have the following components: vestibular membrane, stria-vascularis, and Organ of Corti. The Organ of Corti contains basilar membrane, sensory receptors nearly 23,500 cells overlaid by a gelatinous tectorial membrane. Membrane labyrinth contains endolymph and bony labyrinth contains perilymph. ${ }^{6}$

The labyrinthine artery is a branch of the anterior-inferior cerebellar artery which is a branch of the basilar artery. The basilar membrane is wider and elastic at the apex, vibrates for higher frequency; it is smaller and rigid at the base and vibrates for lower frequency. The cochlea has a tontopic distribution. ${ }^{6}$

The central process of first-order neurons enters the pons at cerebellopontine angle and synapses with second-order neuron dorsal and ventral cochlear nuclei. These second-order neurons cross the pons as trapezoid body intermingled with medial lemniscus and synapses with third-order neuron bodies-medial geniculate body. From there, third-order neurons ascend through the posterior limb of the internal capsule to the temporal lobe. Thus, the potential electrical impulses are coded and relayed to the brain through above nerve root and analyzed in the temporal lobe ${ }^{6}$ (-Fig. 1).

\section{Physiology}

The auditory system acts as a channel and transducer that converts sound pressure waves into electrophysiologic signals that can be interpreted by the higher cortical center of the brain. The cochlea is the site of second transduction. The vibration of the oval window (stapes footplate) causes the vibration of perilymph and basilar membrane of the cochlea. This, in turn, causes movement of the stereocilia of the hair cells resulting in increased permeability of the hair cells to potassium and causing depolarization of the hair cells. ${ }^{7}$

The depolarization results in the secretion of neurotransmitter (glutamate) on to the afferent endings of the cochlear nerve and sensitizing the N-methyl-D-aspartate (glutamate receptors) with resulting electrophysiological neuronal signal. These neuronal signals are analyzed by the cognitive center of the brain. There may be reduction in the numbers of neurons in cochlear nuclei and auditory center of the brain and also reduction in the size of cells and changes in neurochemical makeup. This leads to an inability of the central auditory system to process the sound. ${ }^{7}$

\section{Classification of Sensorineural Loss}

Schuknecht and Gacek ${ }^{8,9}(1964)$ proposed four types of sensorineural hearing loss (-Fig. 2):

1. Sensory-a high frequency hearing loss within speech frequency caused by missing outer hair cells.

2. Neural-loss of cochlear neurons causing progressive loss of speech discrimination in the presence of stable pure tone threshold.

3. Strial-metabolic and vascular degeneration in stria vascularis. Slow progressive hearing loss with up and down curve of audiogram and good speech discrimination.

4. Conductive-changes in the stiffness and properties of the basilar membrane, an evenly sloping hearing loss (linear descending pattern of audiogram).

To add to the above types of sensorineural hearing loss:

5. Mixed-combination of above or mild-to-moderate high frequency loss.

6. Indeterminate-no pathological correlation flat or abrupt high-frequency hearing loss. 


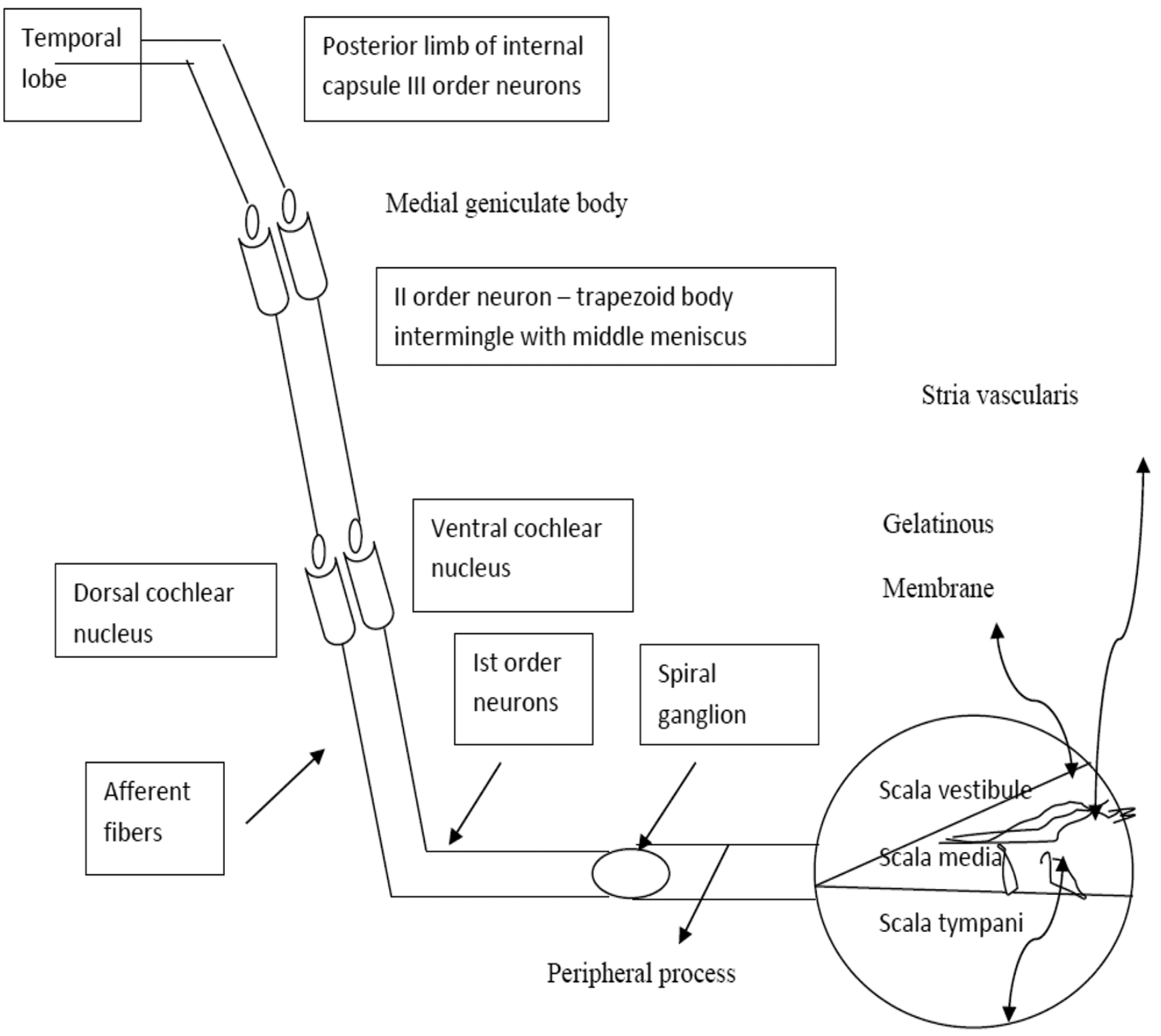

Organ of corti hair cells

Fig. 1 Auditory pathway schematic representation.

\section{Etiology}

Although ageing process causes degeneration of auditory system, other factors are noise and ototoxic drugs, that is, aminoglycosides (cochlear outer hair cells and vestibular cells) diuretic, beta-blockers (reversible hearing loss), Meniere's disease, otosclerosis can affect cochlea. ${ }^{10}$

Systemic diseases such as hypertension, plasma viscosity, atherosclerosis, hyperlipidosis, metabolic bone diseases, diabetic mellitus, hypothyroidism, and Alzheimer's disease ${ }^{11,12}$ are contributing factors to hearing loss, leading to free radical damage, mitochondrial injury, depletion of glutathione and immune inflammatory reactions. Genetic inheritance in hearing loss by mitochondrial DNA involvement has limited evidence. ${ }^{13,14}$

\section{Glutathione}

Glutathione is often referred to as the mother of all antioxidants. Glutathione is produced and used by every single cell in the human body and has a very wide range of scientifically proven health effects. Glutathione is a peptide containing three important amino acids that are glutamate, cysteine and glycine. Cellular glutathione levels are a great predictor of life expectancy; it prevents damage to important cellular components by removing oxygen radicals from the body; thus, it protects many different body systems from diseases and deterioration. It is a defensive agent against the action of toxic xenobiotic (drugs pollutants, carcinogens).

So, it is essential to prevent glutathione levels from becoming low, glutathione deficiency manifest in increased 


\section{Sensory pattern Hearing Loss}

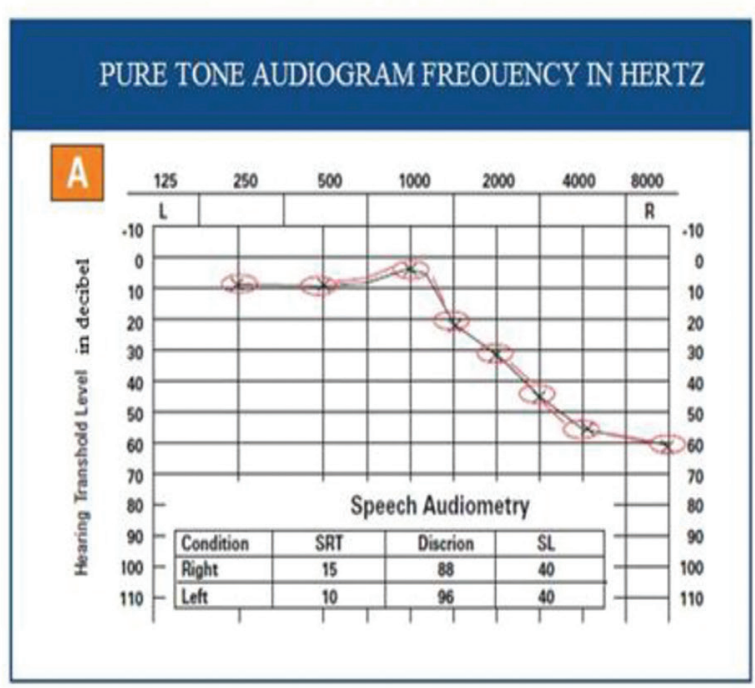

Neural Hearing pattern

\section{PURE TONE AUDIOGRAM FREOUENCY IN HERTZ}

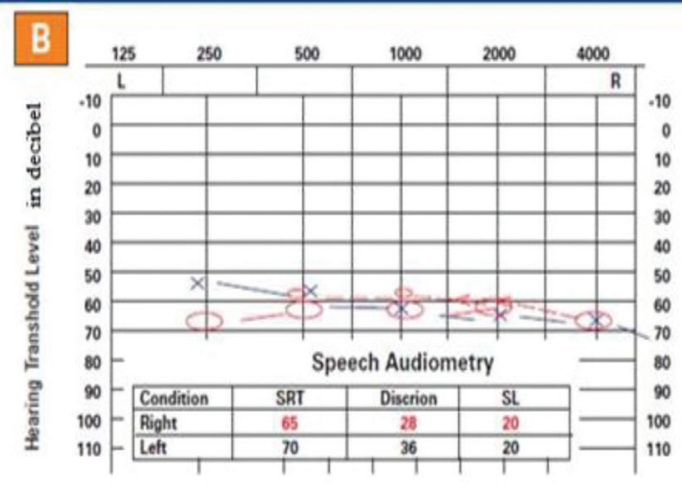

Fig. 2 (A-C) Pattern of hearing loss.

susceptibility to oxidative stress. Glutathione reduces the amount of reactive oxygen species and oxidative stress in the body that would otherwise cause damage to cell and DNA. It is important for the regeneration of other antioxidants such as vitamin C. Glutathione inhibits nuclear factor- $\mathrm{KB}(\mathrm{NF}-\mathrm{kB})$, a transcription factor, that increases the transcription of various inflammatory genes. By inhibitor inflammatory cytokines, it may control the inflammation.

The cells of the human brain consume about $20 \%$ of oxygen utilized by the body, but make up $2 \%$ of the body weight reactive oxygen species is continuously generated during oxidative metabolism; therefore, the detoxification of reactive oxygen species as an essential task within the brain glutathione plays a key role in this process glutathione that may limit neurodegeneration.

Glutathione depletion is a key event that leads to the activation of apoptosis. S-glutathionylation is important for protein modulation and apoptotic (cell death) imitation. Cells depleted of glutathione are susceptible to damage from arachidonic acid. Taking glutathione with vitamin C may

\section{Strial Pattern hearing loss}

\section{PURE TONE AUDIOGRAM FREOUENCY IN HERTZ}

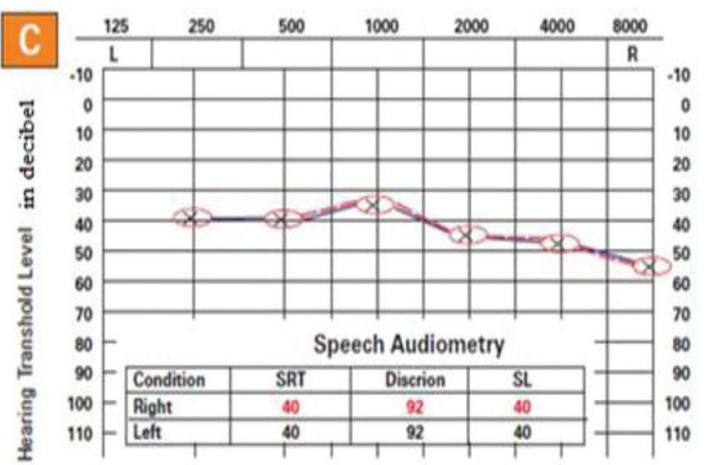

increase its absorption. The changes in the cochlea with other several interacting processes can cause significant effects in hearing.

Added to this noise-induced cause, nuclear, mitochondrial injury, depletion of glutathione (endogenous antioxidant) plays a major role in sensorineural loss. Restoring normal vitamin level can inhibit glutamate release and reduce the oxidative damage and inflammation ${ }^{15}$ ( - Fig. 3).

\section{Management}

Sensorineural hearing loss occurs over a long span of time or sometimes sudden in nature. Hearing aid use may not overcome all hearing problems. However, patients who do not tolerate hearing aids, ear implants may provide improvement in sound quality and clarity. However, the cost is a major limitation for widespread use.

The main therapeutic intervention is to reduce the cochlear damage, inhibit processes or pathways that lead 
Noise related injury

Oxidative stress free radicals

(Nitric oxide peroxynitrite)

Acute \& chronic inflammation
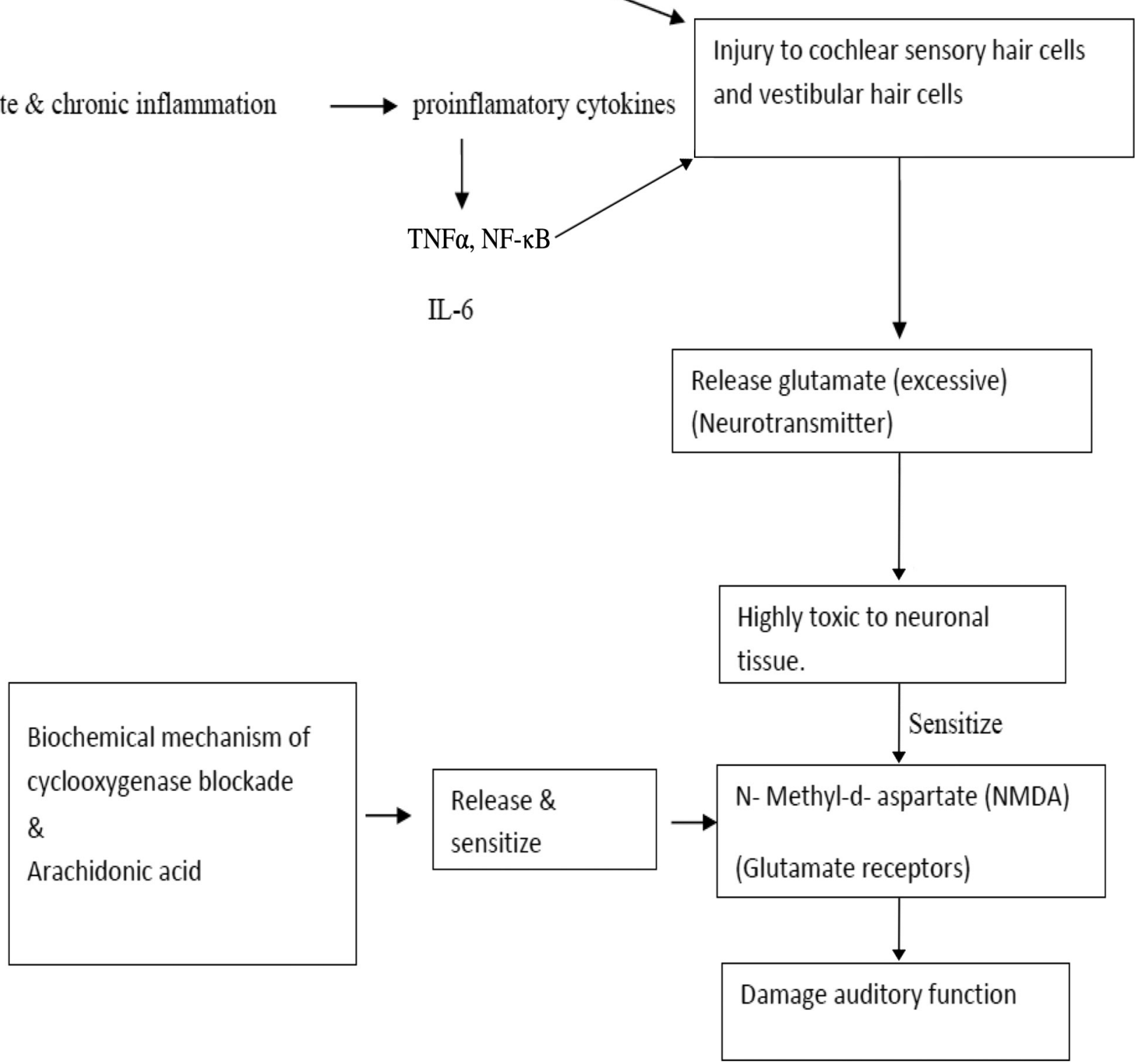

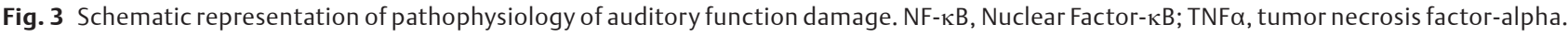

to the damage of cochlear cells and enhance processes that enhance cochlear cell survival.

The drugs used in hearing disorders are as follows:

1. Dextran 40 with betahistine, heparin, pentoxifylline

2. Systematic steroids

3. Antiepileptics

4. Allopurinol

5. Antidepressants

6. Rebamipide
7. Glutamate and N-methyl-D-aspartate (NMDA) receptors antagonists MK-801 ifenprodil, memantine

8. Phospholipids

9. Ginkgo biloba

10. Nonsteroid anti-inflammatory drugs

Antioxidant micronutrients may neutralize free radicals, reduce inflammation, and decrease the effect of glutamate toxicity. ${ }^{16}$ 
Selection of proper type of treatment combinations should be done in terms of neuroprotective effect in hearing disorders as well as restoring normal vitamin levels which can inhibit glutamate release, reduce oxidative damage and inflammation. Vitamin E, alpha lipoic acid, n-acetylcysteine, can protect the inner ear more effectively. A broad spectrum of antioxidants is necessary to address variable free radical induced hearing injury.

Rebamipide, $\mathrm{N}$ acetylcysteine, and alpha lipoic acid antioxidant combination acts by reducing cochlear damage and enhancing cochlear cell preservation.

\section{Rebamipide ${ }^{17,18}$}

- It is mucoprotective agent

- Increases the soluble mucus

- Increases mucosal blood flow

- Inhibitory synthesis of inflammatory cytokines

- Free radicals scavenging effect on reactive oxygen species

Thus, it is an effective anti-inflammatory agent, potent free radical scavenges, and possesses all preservation action.

\section{$\mathrm{N}$-Acetylcysteine ${ }^{19,20}$}

- Increase levels of glutathione the body antioxidant

- Detoxifying, xenobiotic, peroxide compound, and other free radical generating molecules

- Ameliorate the inflammation

- Profound protective effect on cells

\section{Alpha Lipoic Acid ${ }^{21}$}

- Serves a critical role in mitochondrial energy metabolism

- Biological antioxidant a detoxification agent, an important modulator of various inflammatory signaling pathways

- It helps in the synthesis of endogenous antioxidants

- -Increases intracellular glutathione.

Rebamipide along with other antioxidants combination alpha lipoic acid and vitamin $\mathrm{E}$ improves the hearing level at all frequency. Alpha lipoic acid and $\mathrm{N}$-acetylcysteine reduce mitochondrial reactive oxygen species production, thus, reducing high-frequency threshold elevator and maintaining sensorineural tissue survival.

\section{Cinnarizine and Dimenhydrinate}

Calcium channel blocker increases blood flow by vasodilatation and suppresses vestibular cell stimuli.

Steroids plays role as anti-inflammatory agents.

\section{Study Design}

A prospective, noncomparative, metacentric clinical study.

\section{Subjects and Methods}

The study was carried out in 30 patients from May 6, 2016 to January 1, 2018. A total of 30 patients of sensorineural hearing loss were enrolled (17 males, 13 females) ( - Fig. 4A).

\section{Inclusive Criteria}

Healthy males or non-pregnant females of all age group with a diagnosis of sensorineural hearing loss were considered.

\section{Exclusive Criteria}

- Subject with other cause of hearing loss such as otitis media, Meniere's disease, acoustic trauma, acoustic tumor, or otosclerosis.

- The subject who is pregnant, nursing, or planning a pregnancy.

- Subject with complete hearing loss based on pure tone audiometry.

- Patients with a history of schizophrenia, and other psychotic illness.

- Subject with drug-induced ototoxicity and hearing loss.

\section{Overall Study Design}

The total duration of the study was 8 weeks. There were three visits for each patient. After recording the study of disease, the patient had undergone physical and audiogram examination. The patient was administered rebamipide $100 \mathrm{mg}+$ alpha Lipoic Acid $100 \mathrm{mg}$ and acetylcysteine $100 \mathrm{mg}$ capsules twice a day for a total period of 8 weeks.

Wherever giddiness was an added symptom, Cinnarizine $20 \mathrm{mg}$ + Dimenhydrinate $40 \mathrm{mg}$ twice a day was added up to complete relief of symptoms twice daily thereafter once a day as maintenance over a period of 8 weeks. Wherever tinnitus was added symptom, deflazacort $6 \mathrm{mg}$ twice a day added and tapered up to 1 month. If the symptom, tinnitus, persists, intratympanic steroid is injection given.

The patient had to make a visit on week 4 and week 8 . The patient was subjected to physical examination in these visits. Last visit audiogram examination was repeated and clinical assessment was done.

\section{Results}

The auditory system acts as a channel and transducer. ${ }^{22}$ The sound waves from the external environment are channeled through the external auditory canal to the tympanic membrane. The external ear acts as an acoustic resonator to intensify in the range of 2 to $5 \mathrm{kHz}$; those frequencies that are most important in speech. At tympanic membrane first transduction takes place, that is, sound waves convert into vibrative mechanical energy (sound pressure wave) and also amplification of pressure wave takes place in the middle ear. The cochlea was the site of the conversion of mechanical energy to electrophysiologic signal vibration of oval window that causes vibrations of the basilar membrane of the cochlea. This, in turn, causes movement of the stereocilia of the hair cells lying on the basilar membrane resulting in an increase in permeability of hair cells to potassium and depolarization of the hair cells. The depolarization results in the secretion of a neurotransmitter-glutamate onto the afferent endings of the cochlear nerve causing chemical reaction with the glutamate receptor-N-methyl-D-aspartate, with resulting electrophysiologic neuronal signals. The neuronal signals 

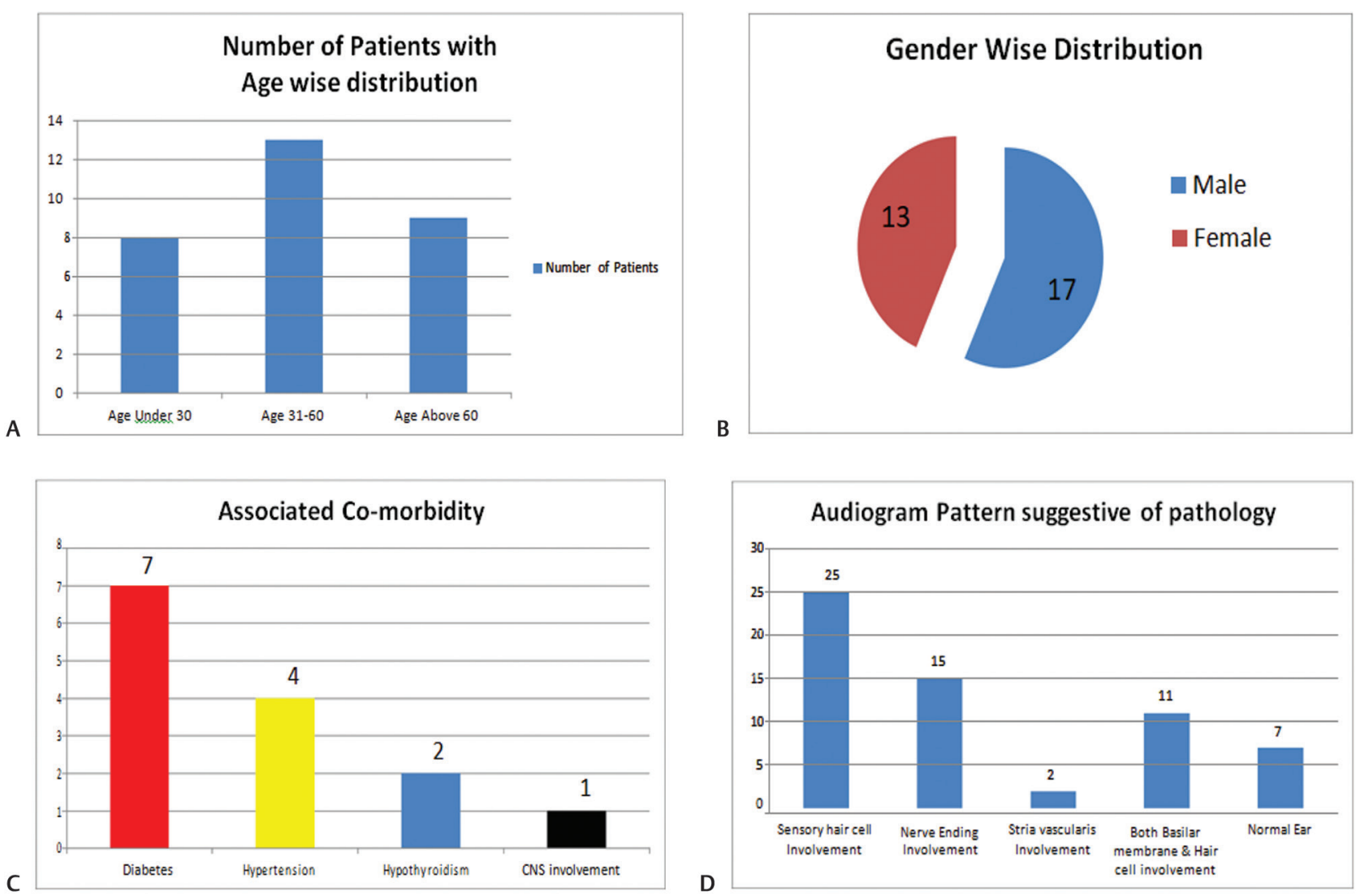

Fig. 4 Statistical analysis of study group data. (A) Number of patients with age-wise distribution. (B) Gender wise distribution. (C) Associated co-morbidity with presentation of hearing loss. (D) Audiogram pattern suggestive of pathology.

are analyzed by cognitive center of the brain to permit an understanding of contents in association with memory. The changes in cochlear hair cells in turn neurochemical makeup cause significant effects on the hearing of person. ${ }^{23}$

The sensorineural hearing loss was synonymous with presbyacusis, but in our study of 30 patients, nearly 8 patients come under the age group of within 30 years and another 13 patients under 31 to 60 years, and 9 patients above 60 years. This was a most distressing factor, causing them handicap in their working place ( - Fig. 4B).

The auditory system is affected by various risk factors such as free radicals damage, mitochondrial injury, excessive secretion of glutamate, NMDA sensitization, depletion of glutathione and immune-inflammatory reaction. Although the ageing process causes degeneration of auditory system other factors such as noise, ototoxic drugs, and infection can cause decline in hearing. ${ }^{24}$

In our study, nearly 27 patients were unable to explain the cause for hard of hearing, only one patient had exposure to noise and two for infection (enteric fever).

Above etiological factors cause injury to the auditory apparatus including sensory and vestibular hair cells. Probably free radical damage was a responsible biochemical factor in the pathogenesis. Levels of the potential toxic glutamate, NMDA receptor, and multiple free radicals were markedly elevated in patients with tinnitus. Depletion of glutathione and ageing were contributing factors to this common pathway of damage.

In our study, all 30 patients all had complaints of hard of hearing; nearly 14 patients had tinnitus and 7 patients had complaints of giddiness. Most distressing factor was a delay in approaching for medical assistant as nearly 15 patients took 6 months to approach us for treatment.

Numerous systemic diseases such as hypertension, plasma hyperviscosity, atherosclerosis metabolic bone disease, diabetic mellitus, hypothyroidism, and Alzheimer's disease were contributing factors to sensorineural hearing loss.

In our study, seven patients were suffering from diabetic mellitus, four patients were of hypertensive, two patients from hypothyroidism and all were under treatment, while one patient computed tomographic scan shows ischemic changes in brain parenchyma. Nearly 23 patients had involvement of both ear, 4 patients had only left ear involvement, and 3 of the right ear only ( - Fig. $4 \mathbf{C}$ ).

There are different types of audiogram patterns noted such as sloping with abrupt steep and progressive at high frequency, parabolic, flat, up and down and slopping to flat, depending on audiogram pattern the disease is classified and the site of pathology is identified.

Depending on audiogram curve, nearly 25 ears have sensory hair cells involvement, 15 ears nerve ending and neural cells, 2 ear stria vascularis, 11 ears were having both basilar 
membrane, hair cells involvement, and 7 ears were of normal in nature. This needs further extensive study ( - Fig. 4D).

\section{Evaluation of Hearing after Treatment}

Determination of hearing was based on pure tone hearing levels at 250, 500, 1,000, 2,000, 4,000, and $8000 \mathrm{KHz}$ and was accomplished by comparing the pre-treatment hearing level with that after 8 weeks of treatment.

The proportion of subjects with improved, unchanged, or worsen on week 8 of treatment analyzed is as follows:

- Improved-Decrease of $\geq 10$ decibels (dB) from baseline score was deemed to denote a clinically significant improvement.

- Unchanged-Decrease or an increase in hearing score but not more than $10 \mathrm{~dB}$ from baseline score represents no change.

- Worsen-Increase of $10 \mathrm{~dB} \geq$ from baseline score (pre-treatment score) indicates deterioration.

In our study, totally 30 patients participated regularly. Out of 60 ears, 7 ears were having normal hearing. Out of remaining 53 ears, 5 ears $(2+2+1)$-of two patients of both ears had hard of hearing and one patient had pathology in right ear)-were rejected because of side effects like vomiting in two cases and acne, skin rashes in one case. So, total 48 ears having hard of hearing were analyzed after 8 weeks of treatment, that is, 21 patients having both ear pathology, and 6 patients were having single ear pathology (4 left ears and 2 right ears). Total 22 ear show improvement, that is, more than $10 \mathrm{~dB}$ decrease from baseline score. Total 26 ear show unchanged or minimal improvement, that is, there was decrease or an increase in hearing score but not more than $10 \mathrm{~dB}$ baseline score. None have worsening of the condition, i.e., increase of more than $10 \mathrm{~dB}$ from baseline score indicating that there were no deteriorated ears ( - Fig. 5A-D), - Table $\mathbf{1}$ ).

\section{Other Symptoms}

Giddiness had been controlled in all seven patients. Tinnitus improved in eight patients (in two patients after injecting intratympanic steroids). In six patients, tinnitus was persisting (in two cases intensity of tinnitus reduced and in four cases no change was noted).

\section{Discussion}

Sensorineural hearing loss affects millions of people is characterized by a decline of auditory function. ${ }^{1}$

The etiology of sensorineural hearing loss is multifactorial, where oxidative stress, free radicals, inflammation of cells of auditory apparatus, mitochondrial damage, excessive secretion of glutamate and its toxicity, depletion of glutathione enzyme have a significant role in the development of sensorineural hearing loss. Other risk factors are noise exposure ototoxic drugs, and health comorbidities., ${ }^{2,3}$

The most of pathological and biochemical changes occur in the cochlea with several interacting processes. A meaningful addition to current otology practice should be based on understanding the synthesis of free radicals in the inner ear and their effect over mitochondrial function, in turn
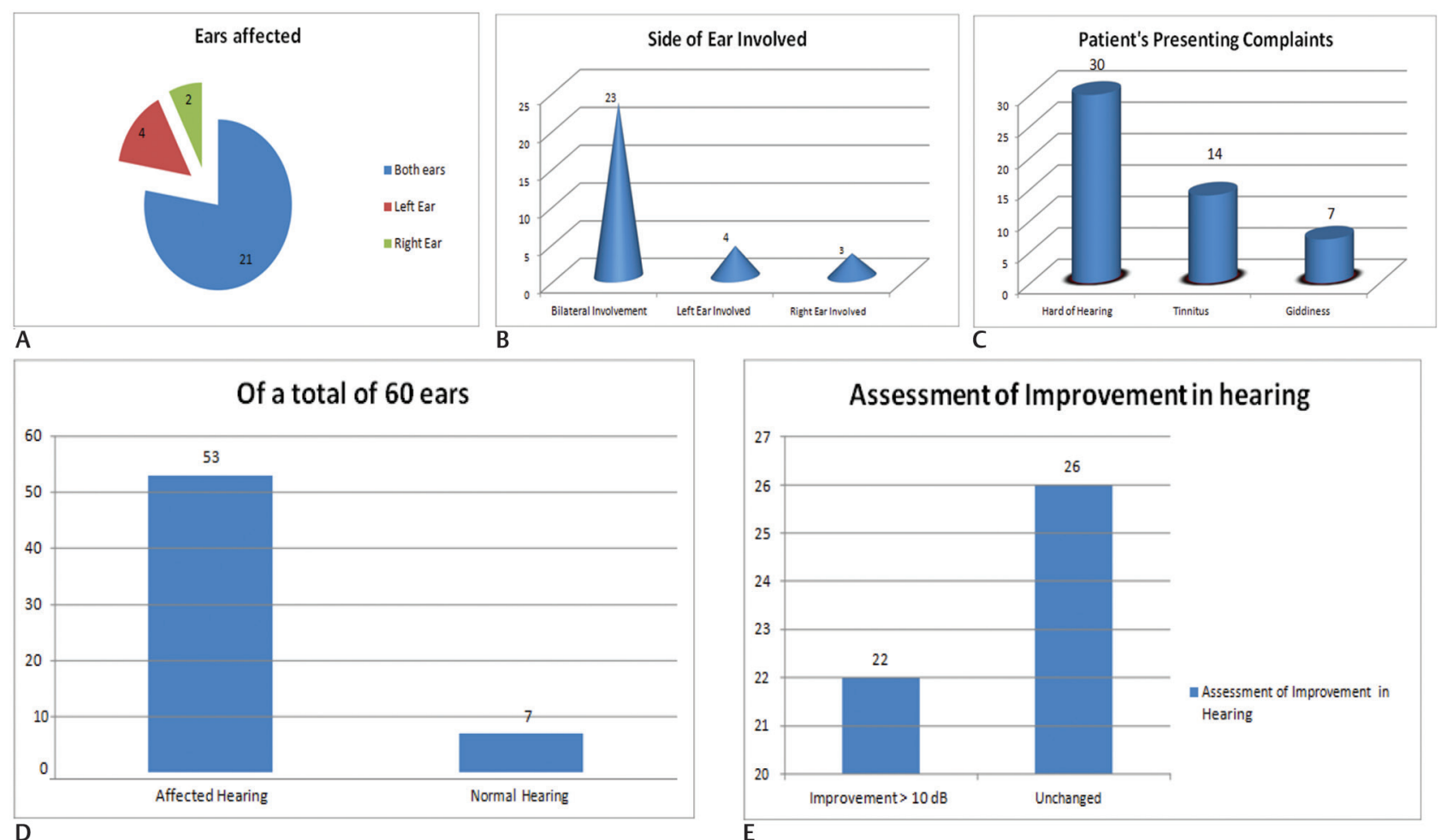

C

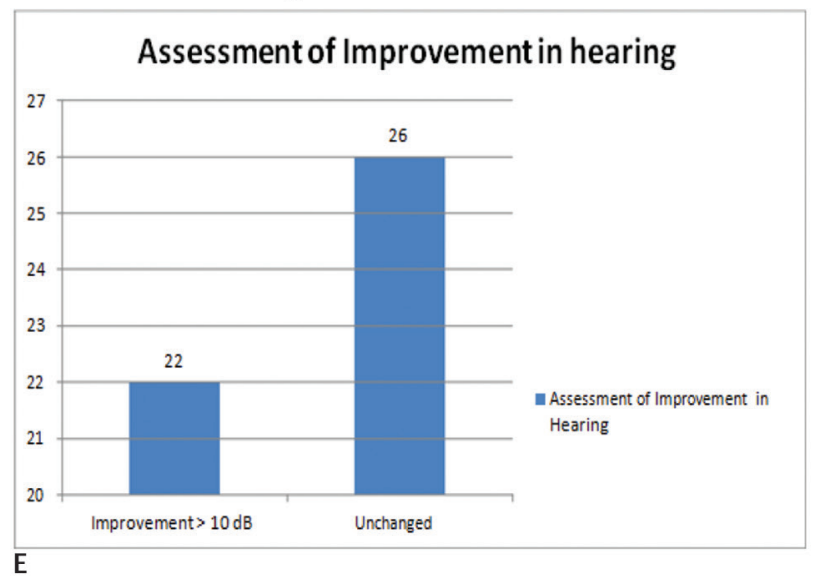

Fig. 5 Statistical analysis of results of study group. (A) Ears affected; after application of exclusion factor, 48 ears were included in the study. (B) Side of ear involved. (C) Patients presenting with complaints. (D) Patient's hearing status. (E) Assessment of Improvement in Hearing after treatment. 
Table 1 Assessment of improvement in hearing after treatment

\begin{tabular}{|c|c|c|c|c|c|c|c|c|c|c|c|c|}
\hline \multicolumn{7}{|c|}{ Mean improvement in the right ear (in decibels) } & \multicolumn{6}{|c|}{ Percentage of improvement in the right ear hearing loss } \\
\hline & 250 & 500 & 1,000 & 2,000 & 4,000 & 8,000 & 250 & 500 & 1000 & 2000 & 4000 & 8000 \\
\hline $\begin{array}{l}\text { Before } \\
\text { treatment }\end{array}$ & 41.5 & 45.625 & 53.125 & 58.125 & 63.125 & 69.375 & 6.65 & 6.85 & 16.47 & 15.00 & 11.88 & 17.1 \\
\hline $\begin{array}{l}\text { After } \\
\text { treatment }\end{array}$ & 38.75 & 42.50 & 44.375 & 49.375 & 55.625 & 57.500 & & & & & & \\
\hline Difference & $2.75 \mathrm{~dB}$ & $3.125 \mathrm{~dB}$ & $8.750 \mathrm{~dB}$ & $8.750 \mathrm{~dB}$ & $7.50 \mathrm{~dB}$ & $11.875 \mathrm{~dB}$ & \multicolumn{6}{|c|}{ In higher frequencies, it is $14.66 \%$} \\
\hline \multicolumn{7}{|c|}{ Mean improvement in the left ear (in decibels) } & \multicolumn{6}{|c|}{ Percentage improvement in the left ear hearing loss } \\
\hline & 250 & 500 & 1,000 & 2,000 & 4,000 & 8,000 & 250 & 500 & 1000 & 2000 & 4000 & $8000 \mathrm{~Hz}$ \\
\hline $\begin{array}{l}\text { Before } \\
\text { treatment }\end{array}$ & 45 & 48.5 & 55.5 & 60 & 67 & 77 & 6.66 & 9.27 & 11.71 & 14.17 & 16.56 & 23.37 \\
\hline $\begin{array}{l}\text { After } \\
\text { treatment }\end{array}$ & 42 & 44 & 49 & 51.5 & 56 & 59 & & & & & & \\
\hline Difference & $3 \mathrm{~dB}$ & $4.5 \mathrm{~dB}$ & $6.5 \mathrm{~dB}$ & $8.5 \mathrm{~dB}$ & $11 \mathrm{~dB}$ & $18 \mathrm{~dB}$ & & & & & & \\
\hline \multicolumn{7}{|c|}{$\begin{array}{l}\text { Mean improvement is better in the left ear. Frequency wise, it was better with } \\
\text { higher frequency ( } 10 \mathrm{~dB} \text { or more) }\end{array}$} & \multicolumn{6}{|c|}{$\begin{array}{l}\text { In higher frequency, it was } 18 \% \text {. So, in our study, better } \\
\text { hearing improvement in higher frequencies was noticed }\end{array}$} \\
\hline
\end{tabular}

biological changes that take place in hair cells and neural cells (excessive secretion of glutamate and its toxic effect over N-methyl-D-aspartate and decreased glutathione and glutathione-S enzymes in hair cells and neuronal cells). ${ }^{24}$

With respect to the above-established mechanisms, a potential treatment strategy that restores normal antioxidant level, inhibits glutamate release and reduces its toxicity and reducing oxidative damage, with decrease in free radicals and inflammation, increasing glutathione levels should be evaluated.

This approach may be also relevant to the patient with tinnitus and giddiness along with hard of hearing. Rebamipide $100 \mathrm{mg}+$ alpha lipoic acid $100 \mathrm{mg}+$ acetylcysteine $100 \mathrm{mg}$ along with combination of cinnarizine $20 \mathrm{mg}$, and dimenhydrinate $40 \mathrm{mg}$ and steroids may be a clinically relevant approach to treat age-related and other sensorineural hearing loss.

- Eight weeks BD dose improves nearly $10 \mathrm{~dB}$ loss.

- This combination will address both inhibiting cochlear cell damage and enhance cochlear cell preservation by reducing free radicals-prevent mitochondrial injuriesdecrease glutamate secretion and its toxicity and enhancing glutathione levels in hair cells.

\section{Conclusion}

Synthesis of free radicals in the inner ear may play an important part in the pathogenesis of sensory hearing loss. Our study demonstrated greater improvement in hearing at higher frequencies with 8 weeks of rebamipide $100 \mathrm{mg}+$ alpha lipoic acid $100 \mathrm{mg}+$ acetylcysteine $100 \mathrm{mg}$ administration in 30 patients with twice-daily dosing.

Present scientific experience supports above combination therapy as effective prophylaxis in sensorineural hearing loss. This combination will address both inhibiting the cochlear cell damage and enhancing cochlear cell preservation.

\section{Ethical Approval}

Informed consent was obtained from all individual participants included in the study. All procedures performed in studies involving human participants were in accordance with the ethical standards of the institutional and/or national research committee and with the 1964 Helsinki Declaration and its later amendments or comparable ethical standards.

\section{Conflict of Interest}

None declared.

\section{Acknowledgements}

We would like to thank all patients who had participated in this study and had followed up timely; this encouraged us to share and submit our data and observations for research publication.

\section{References}

1 Yamasoba T, Lin FR, Someya S, Kashio A, Sakamoto T, Kondo K. Current concepts in age-related hearing loss: epidemiology and mechanistic pathways. Hear Res 2013;303:30-38

2 Wong AC, Ryan AF. Mechanisms of sensorineural cell damage, death and survival in the cochlea. Front Aging Neurosci 2015;7:58

3 Fujimoto C, Yamasoba T. Oxidative stresses and mitochondrial dysfunction in age-related hearing loss. Oxid Med Cell Longev 2014;2014:582849

4 Bridgwood A, General Household Survey: Living in BritainResults from the 1998 General Household Survey. London: The Stationery Office Books; 2000

5 Walling AD, Dickson GM. Hearing loss in older adults. Am Fam Physician 2012;85(12):1150-1156

6 Hyman LH, Hyman's Comparative Vertebrate Anatomy. 3rd edition. University of Chicago Press; 1992: 634. ISBN 0-226-87013-8

7 Guyton Aurthur C, Hall John E, The Sense of Hearing. In: Textbook of Medical Physiology. 11th edition. chapter 52, Saunders Co.; 2005;657-658 
8 Merchant SN, Nadol JB Jr, Eds. Schuknecht's Pathology of the Ear. 3rd edition. Vol. 942. Shelton, People's Medical Publishing House; 2010: 142-147

9 Schuknecht HF, Gacek MR. Cochlear pathology in presbycusis. Ann Otol Rhinol Laryngol 1993;102(1 Pt 2):1-161

10 Wright A, Forge A, Kotecha B, Ototoxicity. In: Booth JB, eds. Scott Browns Otolaryngology. Vol. 3. Otology. Oxford: Butterworth Heinemann; 1997: 3/ 20/1-2/20/31

11 Prasad KN, Cole WC, Prasad KC. Risk factors for Alzheimer's disease: role of multiple antioxidants, non-steroidal anti-inflammatory and cholinergic agents alone or in combination in prevention and treatment. J Am Coll Nutr 2002;21(6):506-522

12 Prasad KN. Antioxidants in cancer care: when and how to use them as an adjunct to standard and experimental therapies. Expert Rev Anticancer Ther 2003;3(6):903-915

13 Gates GA, Couropmitree NN, Myers RH. Genetic associations in age-related hearing thresholds. Arch Otolaryngol Head Neck Surg 1999;125(6):654-659

14 Ensink RJ, Camp GV, Cremers CW. Mitochondrial inherited hearing loss. Clin Otolaryngol Allied Sci 1998;23(1):3-8

15 Waly MI, Al-Attabi Z, Guizani N. Low nourishment of vitamin C induces glutathione depletion and oxidative stress in healthy young adults. Prev Nutr Food Sci 2015;20(3):198-203

16 Joachims HZ, Segal J, Golz A, Netzer A, Goldenberg D. Antioxidants in treatment of idiopathic sudden hearing loss. Otol Neurotol 2003;24(4):572-575
17 Kim HK, Kim JI, Kim JK, et al. Preventive effects of rebamipide on NSAID-induced gastric mucosal injury and reduction of gastric mucosal blood flow in healthy volunteers. Dig Dis Sci 2007;52(8):1776-1782

18 Matysiak-Budnik T, Heyman M, Mégraud F. Review article: rebamipide and the digestive epithelial barrier. Aliment Pharmacol Ther 2003;18(1, Suppl 1):55-62

19 Millea PJ. N-acetylcysteine: multiple clinical applications. Am Fam Physician 2009;80(3):265-269

20 Food and Drug Adminstration. (2006). Brian Harvey. Acetadote Package Insert. [PDF]. Available at: https://www.accessdata. fda.gov/drugsatfda_docs/label/2006/021539s004lbl.pdf. Accessed January 10, 2020

21 Shay KP, Moreau RF, Smith EJ, Smith AR, Hagen TM. Alpha-lipoic acid as a dietary supplement: molecular mechanisms and therapeutic potential. Biochim Biophys Acta 2009;1790(10): 1149-1160

22 Howarth A, Shone GR. Ageing and the auditory system. Postgrad Med J 2006;82(965):166-171

23 Allen JD. Human physiology - the basis of medicine. Ulster Med J 2008;77(3):216

24 Ruel J, Chabbert C, Nouvian R, et al. Salicylate enables cochlear arachidonic-acid-sensitive NMDA receptor responses. J Neurosci 2008;28(29):7313-7323 